\title{
Immunocytochemical Evidence That Neurotensin Is Present in Glutamatergic Neurons in the Superficial Dorsal Horn of the Rat
}

\author{
A. J. Todd, R. C. Spike, R. F. Price, and M. Neilson \\ Department of Anatomy, University of Glasgow, Glasgow G12 8QQ, United Kingdom
}

\begin{abstract}
In order to determine whether glutamate is enriched in neurotensin-containing axons in the superficial dorsal horn of the rat spinal cord, we have carried out preembedding immunocytochemistry with an antiserum to neurotensin and then used a postembedding immunogold method with antiserum to glutamate. The immunogold label (corresponding to glutamate-like immunoreactivity) over $\mathbf{4 0}$ neurotensin-immunoreactive boutons in laminae $I$ and II of the lumbar dorsal horn was compared with that over nearby axons that formed asymmetrical or symmetrical synapses. In addition, for $\mathbf{2 0}$ of these boutons, the labeling was compared with that over mossy and parallel fiber terminals (both of which are thought to use glutamate as a transmitter) from sections of cerebellum that had been processed together with those of spinal cord.
\end{abstract}

Glutamate-like immunoreactivity was consistently high over neurotensin-immunoreactive boutons relative to most surrounding profiles. Immunostaining over these boutons was slightly $(11 \%)$ lower than that over matched terminals that formed asymmetrical synapses, but considerably higher than that over the terminals that formed symmetrical synapses. The level of glutamate immunoreactivity in neurotensin-immunoreactive boutons in dorsal horn was similar to that in cerebellar parallel fiber terminals, but significantly lower than that in mossy fiber terminals.

These results suggest that glutamate is a transmitter used by neurotensin-immunoreactive axons in the dorsal horn, and since these axons are thought to be largely or entirely derived from neurotensin-containing neurons in laminae IIII, they provide immunocytochemical evidence for a population of excitatory glutamatergic neurons in this region.

[Key words: glutamate, excitatory neuron, spinal cord, peptide]

The superficial part of the spinal dorsal horn (laminae I-III) contains densely packed small neurons (Rexed, 1952), and many of these have axons that terminate locally (Bennett et al., 1980; Nandi et al., 1993). Immunocytochemical studies have provided evidence that some of these neurons contain the inhibitory transmitter GABA, and that this may coexist with glycine or ACh (Hunt et al., 1981; Barber et al., 1982; Magoul et al., 1987; Todd and McKenzie, 1989; Carlton and Hayes, 1990; Todd and

\footnotetext{
Received May 6, 1993; revised July 21, 1993; accepted July 29, 1993.

We thank Mr. D. Russell, Mr. R. Kerr, Miss C. Morris, and Miss M. Hughes for excellent technical assistance. This work was supported by a grant from the Wellcome Trust.

Correspondence should be addressed to Dr. A. J. Todd at the above address.

Copyright (C) 1994 Society for Neuroscience 0270-6474/94/140774-11\$05.00/0
}

Sullivan, 1990; Maxwell et al., 1991; Todd, 1991). However, these cells only account for approximately $30 \%$ of the neuronal population in laminae I and II, and $46 \%$ of that in lamina III (Todd and Sullivan, 1990), and the majority of neurons in each of these laminae therefore do not appear to contain any of these transmitters.

Although the classical neurotransmitter(s) used by the remaining neurons is not known, there are several lines of evidence that suggest at least some of these cells are excitatory, and use glutamate or a related acidic amino acid. If ${ }^{3} \mathrm{H}-\mathrm{D}$-aspartate is injected into spinal gray matter, it is accumulated by a population of neurons in laminae I-III (Rustioni and Cuenod, 1982), and these cells are not GABA immunoreactive (Antal et al., 1991). It is likely that this uptake mechanism is restricted to neurons that use an acidic amino acid as a transmitter (Streit, 1980). In addition, some neurons with cell bodies in the superficial dorsal horn have been shown to give rise to axons that form asymmetrical synapses (Light and Kavookjian, 1988; Spike and Todd, 1992), and this type of synapse is thought to be generally associated with excitatory transmission. However, synaptic ultrastructure may not be a reliable guide to transmitter content, since there have also been reports of glutamic acid decarboxylase-and GABA-immunoreactive boutons that form asymmetrical synapses in the dorsal horn (McLaughlin et al., 1975; Carlton and Hayes, 1990). There is also a great deal of physiological evidence that dorsal horn neurons receive much of their sensory input through polysynaptic pathways, presumably involving excitatory interneurons (for review, see Woolf, 1989).

It has so far proved difficult to identify glutamatergic neurons in the spinal dorsal horn with immunocytochemistry. When antisera to glutamate are applied to spinal cord sections, immunoreactive cell bodies are seen in the superficial dorsal horn (Weinberg et al., 1987; Miller et al., 1988) and it was concluded in these studies that the immunoreactive cells were glutamatergic neurons. However, there is much evidence that the presence of glutamate-like immunoreactivity (glutamate-LI) in cell bodies is not a reliable marker for neurons that use glutamate as a transmitter, since the cell bodies of neurons that are thought not to use glutamate (including motor neurons and presumed GABAergic and glycinergic neurons) may possess glutamate-LI (Ottersen and Storm-Mathisen, 1984; Yingcharoen et al., 1989; Walberg et al., 1990). It is thought that, unlike the situation with glycine and GABA, the "metabolic pool" of glutamate is sufficiently large that it can be detected in the cell bodies of many neurons (Fonnum, 1984; Yingcharoen et al., 1989).

The examination of glutamate immunoreactivity in axon terminals appcars to be a more reliable way of identifying neurons that use glutamate as a transmitter. When the postembedding 
immunogold technique is used with glutamate antisera, there is an almost linear relationship between gold particle density and glutamate concentration (Ottersen, 1989), and it is therefore possible to compare the relative concentrations of glutamateimmunoreactive material in different profiles. In the cerebellum it has been shown that the level of glutamate-LI is significantly higher in mossy and parallel fiber terminals (which are thought to release glutamate) than in other cellular compartments, including granule cell bodies that give rise to parallel fibers (Somogyi et al., 1986). Elsewhere in the CNS enrichment of glutamate-LI has been demonstrated in axon terminals that are thought to be glutamatergic (e.g., Montero and Wenthold, 1989; Maxwell et al., 1990; Broman and Ottersen, 1992). This suggests that within axon terminals it is possible to identify the "transmiller pool" of glutamate.

A dense plexus of neurotensin-immunoreactive fibers is present in laminae I and II of the dorsal horn, and neurotensinimmunoreactive neuronal cell bodies are located in laminae IIII, with very few present in deeper laminae (Gibson et al., 1981; Hunt et al., 1981; Jennes et al., 1982; Seybold and Elde, 1982; Yaksh et al., 1982; Leah et al., 1988). The neurotensin-immunoreactive cells in these laminae do not appear to project to the brain (Leah et al., 1988), and they are not GABA immunoreactive (Todd et al., 1992). Since dorsal root section and spinal hemisection do not cause a detectable depletion of neurotensin-LI (Ninkovic et al., 1981; Yaksh et al., 1982), immunoreactive axons in laminae I and II are presumably derived (at least mainly) from intrinsic interneurons located in laminae I-III, which do not contain GABA. In this study we have used preembedding immunocytochemistry to identify neurotensinimmunoreactive axons and have then performed postembedding immunostaining to determine whether they are enriched with glutamate-LI. In addition, in some cases we have compared the levels of glutamate-LI in neurotensin-immunoreactive axons in the dorsal horn with those in cerebellar parallel and mossy fiber terminals, since there is strong evidence that these latter types of axon use glutamate as a transmitter (Sandoval and Cotman, 1978; Stone, 1979; Garthwaite and Brodbelt, 1990).

\section{Materials and Methods}

Tissue preparation and preembedding immunocytochemistry. Four adult Albino-Swiss rats of either sex (190-270 gm) were deeply anesthetized and perfused through the left ventricle with a fixative containing $1 \%$ formaldehyde, $1 \%$ glutaraldehyde in $0.1 \mathrm{M}$ phosphate buffer. The L4 spinal segment was removed from each animal, and the cercbcllum was also removed from two animals. The tissue was rinsed in buffer overnight and sectioned on a Vibratome at $60 \mu \mathrm{m}$. The spinal cord segments were cut into transverse sections and the cerebella were cut at right angles to the long axis of the folia. The sections were treated with $1 \%$ sodium borohydride for $30 \mathrm{~min}$ (Kosaka et al., 1986), rinsed extensively in phosphate-buffered saline (PBS), and incubated overnight in rabbit antiserum to neurotensin (Peninsula) diluted to 1:10,000 in PBS containing $1 \%$ goat serum. They were further processed by an immunogoldsilver method (Chan et al., 1990). The sections were rinsed in PBS and incubated for $3 \mathrm{hr}$ in goat anti-rabbit IgG coupled to $1 \mathrm{~nm}$ gold particles (Biocell) diluted 1:100 in PRS containing 1\% goat serum. They were then rinsed in PBS, postfixed for $30 \mathrm{~min}$ in $2 \%$ glutaraldehyde, rinsed thoroughly in PBS and water, and treated with a silver intensification method (IntenSE M, Amersham UK) according to the manufacturer's instructions. The optimal time for the intensification step was found to be between 11 and $15 \mathrm{~min}$ at approximately $20^{\circ} \mathrm{C}$. The sections were then rinsed in water, osmicated, dehydrated in acetone, and flat-embedded in Durcupan between glass coverslips. Control sections of spinal cord were processed in the same way, except that the primary antiserum was treated with synthetic neurotensin (Sigma; $10 \mu \mathrm{g} / \mathrm{ml}$ diluted antiserum) for $1 \mathrm{hr}$ before the reaction.
Postembedding immunocytochemistry. Two or three sections of spinal cord from each rat and one section of cerebellum from two rats were mounted onto resin blocks. Ribbons of ultrathin sections (silver-gold interference color) were cut from spinal cord with a diamond knife and mounted in serial order onto Formvar-coated nickel mesh grids or uncoated copper mesh grids. Approximately four sections were collected on each grid, and nickel and copper grids were used alternately. Ultrathin sections were also cut from cerebellum and mounted onto Formvarcoated nickel grids. Postembedding immunocytochemistry was performed (on those sections on nickel grids) by the method of Holstege (1991) with a rabbit antiserum to conjugated glutamate (Arnel). Briefly, grids were placed for $1 \mathrm{hr}$ on a droplet of PBS containing $0.1 \%$ Triton-X 100 (PBST), stored overnight at $4^{\circ} \mathrm{C}$ on droplets of glutamate antiserum diluted 1:40,000 in PBST, rinsed, and incubated for $2 \mathrm{hr}$ with goat anti-rabbit IgG coupled to $10 \mathrm{~nm}$ gold particles (Biocell) diluted $1: 25$. At the end of the immunoreaction, the sections were lightly stained with lead citrate. Spinal cord sections on copper grids did not undergo the postembedding reaction and were stained with uranyl acetate and lead citrate.

In order to confirm that the secondary antibody was not recognizing neurotensin antibodies from the preembedding reaction, the postembedding reaction was carried out on some grids with omission of glutamate antiserum. Control sections for the postembedding reaction were treated as described above, except that the glutamate antiserum was incubated with glutamate conjugated to bovine serum albumin for $1 \mathrm{hr}$ beforc this was applied to the sections. The conjugate was made by the method of Storm-Mathisen et al. (1983), and $0.1 \mu \mathrm{l}$ was added to $50 \mu \mathrm{l}$ of diluted antiserum $(1: 40,000)$. The glutamate antiserum is reported to show no cross-reaction with aspartate, GABA, $\beta$-alanine, glycine, or glutamine (manufacturer's specification).

To allow for comparison between neurotensin-immunoreactive boutons in dorsal horn and parallel and mossy fiber terminals in cerebellum, all of the grids of spinal cord sections from two of the animals were processed in parallel with grids containing sections of cerebellum (from the same animal). To ensure the minimum possible variation in incubation conditions, in each case a grid of spinal cord and one of cerebellum were processed on the same droplets of immunoreagents and rinsing solutions.

Analysis. Forty neurotensin-immunoreactive boutons were used to obtain quantitative data: 10 from each of the four rats. These were taker from two or three Vibratome sections from each rat, such that no more than five boutons were obtained from a single Vibratome section. The neurotensin-immunoreactive axonal boutons were initially identified on sections from copper grids (which had not been reacted by the postembedding method). In order to confirm that the bouton was neurotensin immunoreactive, it was examined and photographed on at least three sections. Only boutons that contained silver particles on each of these sections and had a minimum of 10 particles were accepted as being immunoreactive. For comparison, in each case the nearest axonal boutons that formed asymmetrical and symmetrical synapses were also identified (again, on sections that had not been reacted with glutamate antiserum). The neurotensin-immunoreactive bouton and the boutons forming asymmetrical and symmetrical synapses were then examined and photographcd on a nearby section that had been reacted with glutamate antiserum. For 20 of these neurotensin-immunoreactive boutons (obtained from seven different grids), corresponding sections of cerebellum that had been processed on the same droplets were also analyzed. For each of the seven grids of cerebellar tissue, 10 parallel fiber boutons and three mossy fiber terminals were selected at random and photographed. The selection was made at a sufficiently low magnification that gold particles could not be seen.

The densities of gold particles overlying these profiles were analyzed from photomicrographs by means of a digitizing tablet connected to an IBM-compatible computer and viDAs 2.1 image analysis software (Kontron Flectronic, Munich). For the boutons from spinal cord and for cerebellar parallel fiber terminals, the area of the profile was divided by the number of gold particles (for neurotensin-immunoreactive profiles the area occupied by silver particles was subtracted, since these would have obscured overlying gold particles). For mossy fiber terminals, a 2 $\mu \mathrm{m}^{2}$ circle was placed randomly over the terminal three times, and the average of the gold particle densities within these areas was calculated.

To allow comparison between the groups of matched profiles in the dorsal horn, each of the 40 neurotensin-immunoreactive boutons and the boutons forming symmetrical synapses were normalized against the corresponding bouton that formed an asymmetrical synapse, by giving 
the latter a value of $100 \%$. The resulting percentages were then converted to logarithms to allow calculation of the mean percentages for each type of profile. In one case, a bouton that formed a symmetrical synapse contained no gold particles, and therefore had a normalized value of $0 \%$ : this bouton was excluded in order to allow an average value to be calculated. The 20 neurotensin-immunoreactive boutons that were to be compared with cerebellar material were similarly normalized against the average gold particle density in the 10 parallel fiber and three mossy fiber terminals that were examined on the equivalent grid, and the results were treated in the same way.

\section{Results}

\section{Neurotensin immunoreactivity}

When the Vibratome sections of dorsal horn were viewed with the light microscope, neurotensin-LI was seen as small black profiles in laminae I and II and occasional gray cell bodies located on either side of the border between laminae II and III (which could be identified because of the absence of myelin from the ventral part of lamina II). The immunostaining was clearly restricted to the most superficial parts of the sections. Immunostaining was completely absent in sections that had been incubated in primary antiserum that had been treated with neurotensin.

Electron microscopy revealed that silver particles of varying sizes were specifically concentrated over certain axonal boutons in laminae I and II (Figs. 1-3). There was also accumulation of silver particles in occasional large dendrites and neuronal cell bodics, particularly near the border betwecn laminac II and III. No labeled axonal boutons were seen in lamina III. A few silver particles were scattered over the neuropil; however, this background could easily be distinguished from specific labeling since silver particles were not present over the same profiles in serial sections. The density of silver particles was greatly reduced in tissue that was located more than a few micrometers from the original surface of the Vibratome section.

Neurotensin-immunoreactive boutons varied in size and shape. The mean cross-sectioned area of the 40 boutons used for quantitative analysis was $0.99 \mu \mathrm{m}^{2}$ ( $\left.\pm 0.06 \mathrm{SEM}\right)$. They contained many agranular vesicles, which were usually round and which often filled the profile. Dense-cored vesicles were generally also present, but in small numbers $(0-5 /$ profile in a single section), although a few boutons contained considerably more of these vesicles (Fig. 1d). The dense-cored vesicles were often present at the periphery of the profile (Fig. $2 a-c$ ), and were never seen to be clustered near active sites of synapses. Silver particles were frequently associated with dense-cored vesicles, although they were often independent of them (however, large particles may have obscured underlying dense-cored vesicles). In several cases, the immunoreactive boutons were presynaptic to dendrites (Fig. 1c,d) and sometimes to cell bodies (Fig. 1e,f). Synaptic specializations were generally of the asymmetrical type (Fig. $1 c, f$ ) but were occasionally symmetrical (Fig. 1d).

\section{Glutamate immunoreactivity}

The absolute densities of $10 \mathrm{~nm}$ gold particles (corresponding to glutamate-LI) in sections of dorsal horn varicd considerably between different grids. However, in all cases the gold particle density was particularly high over many axons that contained round vesicles. These were often seen to form asymmetrical synapses and sometimes constituted the central axons of synaptic glomeruli. The density of gold particles was low over profiles that contained flattened vesicles or formed symmetrical synapses (Fig. $2 d$ ) and was variable over other structures (dendrites and cell bodies). The neurotensin-immunoreactive boutons were always enriched with gold particles, compared to most of the surrounding structures (Figs. $2 d, 3 a$ ). The densities of gold particles over the 40 neurotensin-immunoreactive boutons and the corresponding boutons that formed asymmetrical or symmetrical synapses are shown in Figure 4 . Although there was considerable variation in the absolute values, the density of gold particles in neurotensin-immunoreactive boutons was consistently higher than that in the boutons that formed symmetrical synapses, and usually similar to that in the boutons that formed asymmetrical synapses. The density of gold particles in neurotensin-immunoreactive boutons was found to be, on average, $88.9 \%$ of that in the boutons that formed asymmetrical synapses, while the density in the boutons that formed symmetrical synapses was $26.9 \%$ of that in the boutons that formed asymmetrical synapses. The differences between the densities in neurotensin-immunoreactive boutons and those in the other two groups were both significant $(p<0.05$ when compared to "asymmetrical" boutons; $p<1 \times 10^{-7}$ when compared to "symmetrical" boutons; Wilcoxon's signed rank test). The background gold density was estimated by examining the lumina of two capillaries on one section from each grid that was examined. The density of gold particles over the resin in these capillaries was never more than 0.5 particles $/ \mu \mathrm{m}^{2}$.

Within the cerebellum, the density of gold particles was elevated over parallel and mossy fiber terminals (Fig. $3 c, d$ ). The mean densities of particles over the 10 parallel fiber and three

\footnotetext{
Figure 1. Examples of neurotensin-immunoreactive boutons in sections that had not undergone postembedding immunocytochemistry. $a-c$, Three serial sections through a lightly labeled bouton $(A)$ that progressively surrounds a small dendritic profile $(D)$. In each micrograph the bouton contains silver particles, some of which are indicated with arrows. Some of these particles are associated with dense-cored vesicles. In $c$ the bouton forms an asymmetrical synapse with the dendrite $(D)$. $d$, A neurotensin-immunoreactive bouton that contains many dense-cored vesicles forms a symmetrical synapse (arrow) onto a small profile. $e$ and $f$, Adjacent sections through a small neurotensin-immunoreactive axon $(A)$ that forms an asymmetrical axosomatic synapse (between arrows) onto a cell body $(S)$. Part of the nucleus $(N)$ can be seen in $e$. Scale bars, $0.5 \mu \mathrm{m}$.

Figure 2. $a-c$, Three serial sections through a neurotensin-immunoreactive axon $(A)$, that forms an oblique synapse (between arrows) onto a dendrite $\left(D_{1}\right)$. A nearby bouton $(B)$, which contains flattened vesicles, and another dendrite $\left(D_{2}\right)$ are also seen. $d$, A section adjacent to $c$ that had been reacted with glutamate antiserum; $10 \mathrm{~nm}$ gold particles, representing glutamate-LI, are concentrated over the neurotensin-immunoreactive axon $(A)$, and are present at much lower densities over the two dendrites $\left(D_{t}\right.$ and $\left.D_{2}\right)$ and the bouton $(B)$. Scale bar, $0.5 \mu \mathrm{m}$ for $a-d$.

Figure 3. A comparison between the glutamate-LI over a neurotensin-immunoreactive bouton and that over cerebellar parallel and mossy fibers. $a$, A section through a neurotensin-immunoreactive axon $(A)$. The section has been reacted with glutamate antiserum, and 10 nm gold particles (representing glutamate-LI) are more numerous over $A$ than over other structures, including a vesicle-containing profile $(V)$. $b$, An adjacent section through the same neurotensin-immunoreactive axon $(A)$. This section had not been reacted with glutamate antiserum, and the silver particles from the preembedding reaction are clearly seen. $c$, A section through the molecular layer of the cerebellum to show three parallel fiber terminals $\left(P_{I}-\right.$ $P_{3}$ ) forming synapses (arrows). $d$, Part of a mossy fiber terminal $(M F)$, from the granular layer. The section of cerebellum from which $c$ and $d$ are taken was processed on the same droplets of immunoreagents as that from which $a$ is obtained. Note that the gold particle density over the neurotensin-immunoreactive axon is similar to the densities over the parallel fibers, but lower than that over the mossy fiber terminal. Scale bar, $0.5 \mu \mathrm{m}$ for $a-d$.
} 

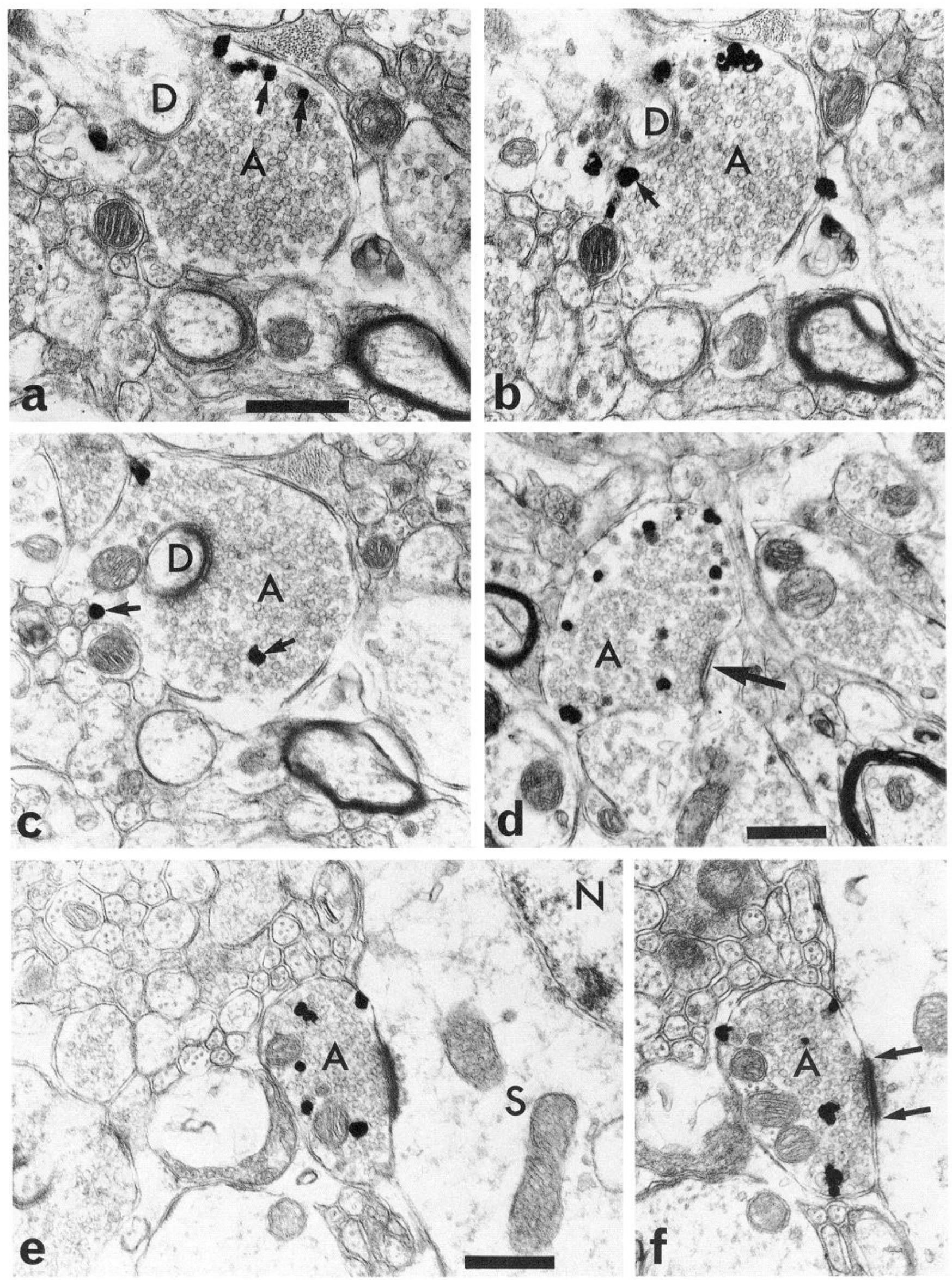

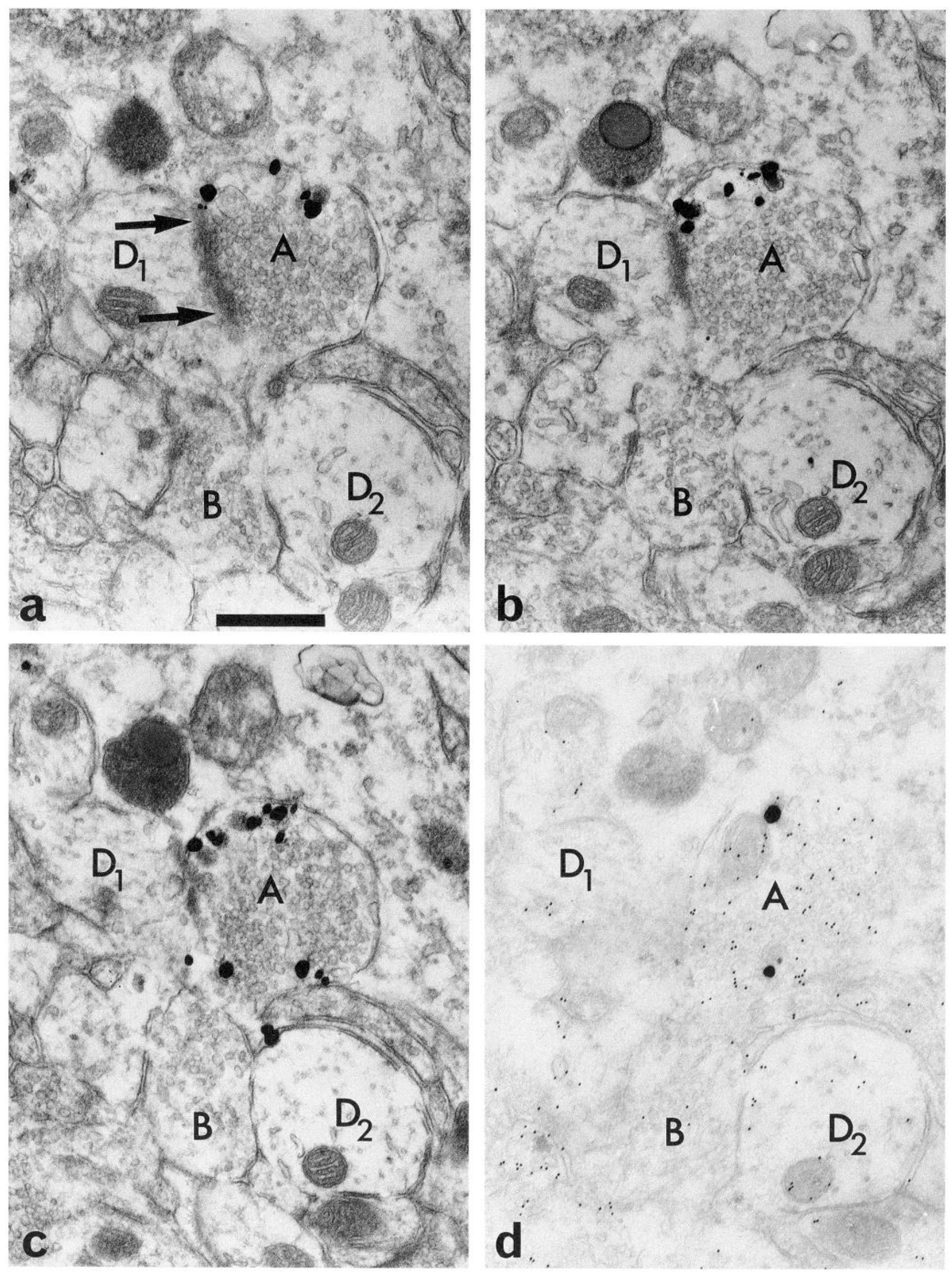


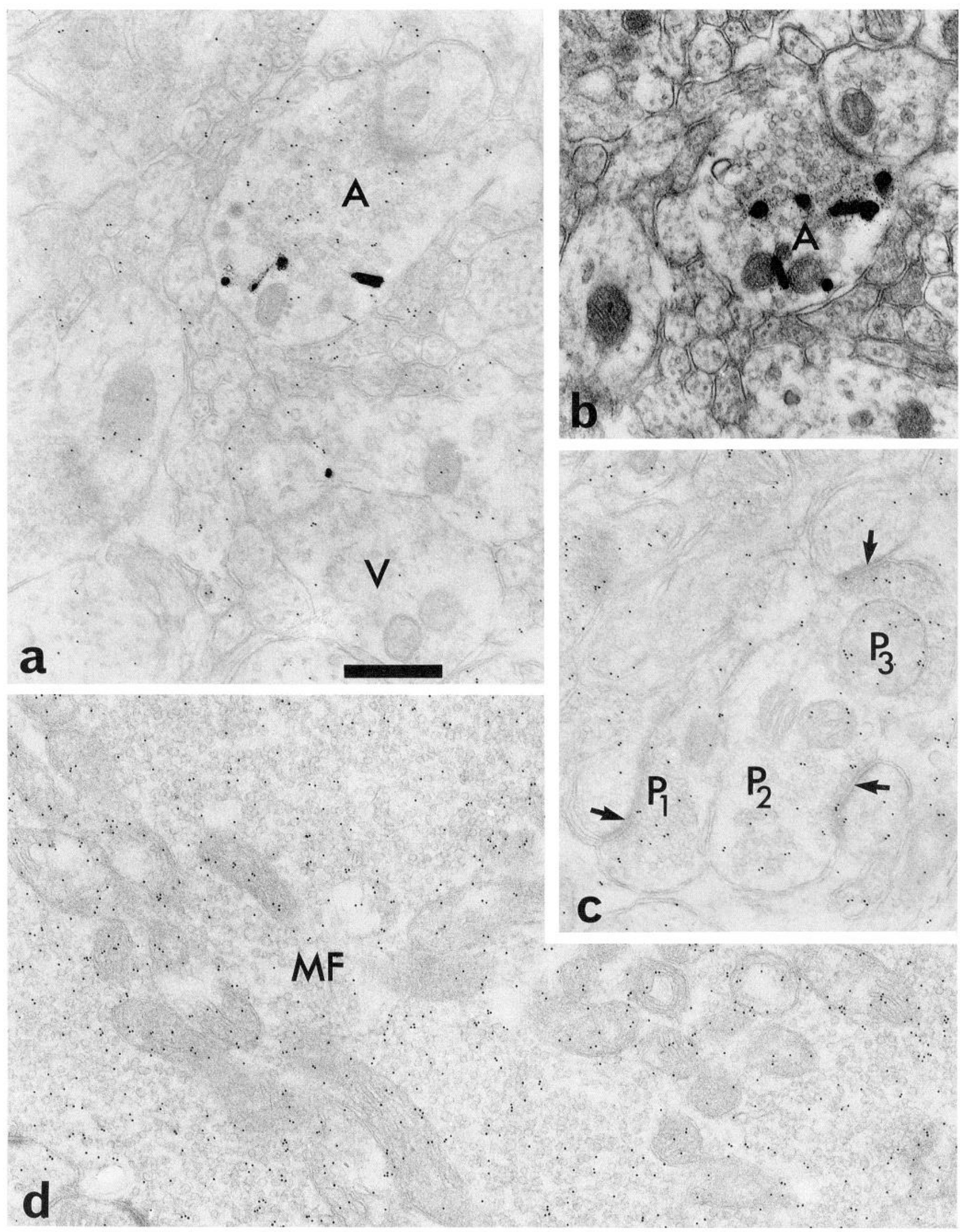




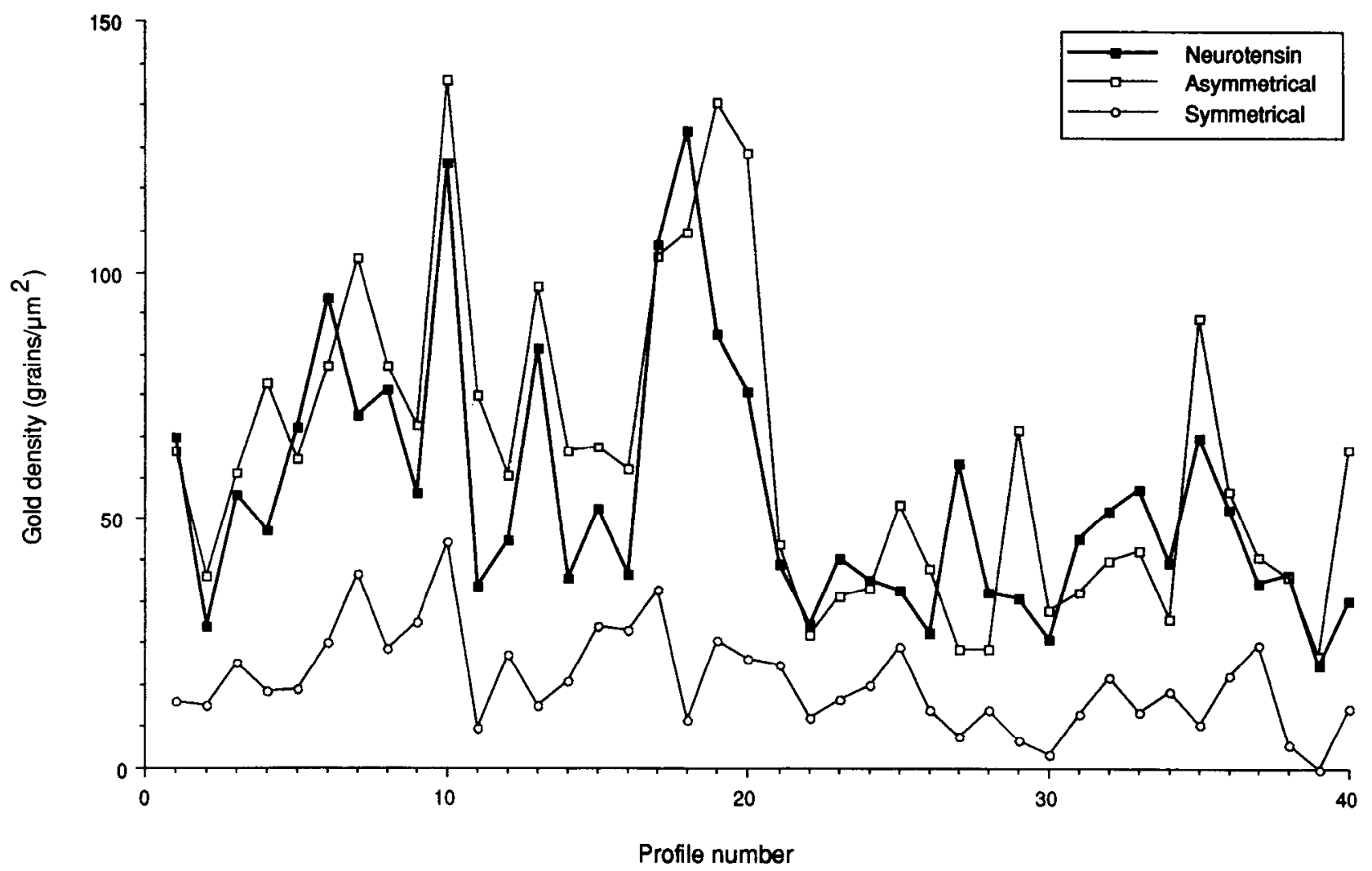

Figure 4. A diagram to show the gold particle density (representing level of glutamate-LI) in the 40 neurotensin-immunoreactive boutons (thick line) and in the matched boutons that formed asymmetrical synapses (upper thin line) and symmetrical synapses (lower thin line). Each number along the abscissa represents three profiles, one of each type. Although there is considerable variation in the absolute density of gold particles, the densities in the neurotensin-immunoreactive boutons are generally similar to those in the boutons that formed asymmetrical synapses, and both are higher than the densities in the boutons that formed symmetrical synapses. Even though the values are not continuously variable, each set of points is joined by lines, in order to facilitate comparison between the gold particle densities of members of each set. Note that boutons numbered from 21 to 40 are those that were compared with equivalent sections of cerebellum.

mossy fiber terminals from each grid are shown in Table 1, together with the identities of the neurotensin-immunoreactive boutons to which they correspond (the bouton numbers are the same as those in Fig. 4). The neurotensin-immunoreactive boutons had a density of gold particles that was $107.4 \%$ of that in parallel fiber terminals and $69.4 \%$ of that in mossy fiber terminals. The difference in gold particle density between neurotensin-immunoreactive boutons and mossy fiber terminals was highly significant ( $p<0.001$, Wilcoxon's signed rank test); however, there was no significant difference between neurotensinimmunoreactive boutons and parallel fiber terminals.

When the postembedding reaction was carried out with glutamate antiserum omitted, no $10 \mathrm{~nm}$ gold particles were seen on the sections (Fig. $5 a$ ). If the primary antiserum was incubated with glutamate conjugated to BSA, the reaction was strongly suppressed and very few particles were observed in any profiles (Fig. $5 b$ ).

\section{Discussion}

One of the major problems in identifying neurons that use glutamate as a transmitter is that, unlike the situation with GABA and glycine, a significant proportion of the glutamate present in the cell bodies and dendrites of neurons may represent a "metabolic pool," and this cannot readily be distinguished from the "transmitter pool" of the amino acid with light microscopic immunocytochemistry. However, there is considerable evi- dence that glutamate is highly concentrated in axonal boutons from which it is released as a transmitter, and that the presence of high levels of glutamate-LI in axon terminals provides one of the most reliable ways of identifying glutamatergic neurons (Somogyi et al., 1986; Montero and Wenthold, 1989; Maxwell et al., 1990; Broman and Ottersen, 1992). By identifying the axons of particular neurons and estimating the level of glutamate-LI in them, it should therefore be possible to determine whether or not the cells use glutamate as a transmitter. However, it has proved difficult to label the axons of neurons whose cell bodies are located in laminae I-III of the spinal dorsal horn. Most of these neurons are small and therefore difficult to impale with intracellular microelectrodes. Many have axons that appear to terminate locally, and cannot be labeled with anterograde transport methods, and their axonal boutons do not form characteristic synaptic configurations that can be recognized in normal maierial.

In the present study we have attempted to get around this problem by using preembedding immunocytochemistry with an antiserum to the peptide neurotensin in order to label axons derived from a particular population of dorsal horn neurons, and then carrying out postembedding immunostaining to detect glutamate-LI on the same tissue. In preliminary studies we found that if an immunoperoxidase method [e.g., the avidin-biotin complex $(\mathrm{ABC})$ method] was used to detect peptide-containing axons, this strongly suppressed subsequent postembedding im- 
Table 1. Gold particle density in cerebellar parallel and mossy fibers

\begin{tabular}{llll} 
Grid & $\begin{array}{l}\text { Equivalent } \\
\text { boutons }\end{array}$ & \begin{tabular}{l} 
Mean gold particle densities \pm SEM \\
\cline { 2 - 4 }$(n=10)$
\end{tabular} & $\begin{array}{l}\text { Mossy fibers } \\
(n=3)\end{array}$ \\
\hline 1 & $21-25$ & $33.84 \pm 2.41$ & $67.17 \pm 2.36$ \\
2 & $26-30$ & $41.63 \pm 2.75$ & $44.67 \pm 5.44$ \\
3 & 31 & $48.77 \pm 5.92$ & $56.67 \pm 3.50$ \\
4 & $32-33$ & $32.20 \pm 3.34$ & $53.56 \pm 9.17$ \\
5 & 34 & $38.16 \pm 4.57$ & $63.22 \pm 3.30$ \\
6 & $35-38$ & $29.21 \pm 3.74$ & $56.33 \pm 9.02$ \\
7 & $39-40$ & $44.85 \pm 6.14$ & $62.17 \pm 4.82$ \\
\hline
\end{tabular}

Data are the densities of gold particles in parallel and mossy fibers that were used for comparison with 20 of the neurotensin-immunoreactive boutons (those numbered $21-40$ in Fig. 4). For each grid, 10 parallel fibers and three mossy fibers were selected at random from a single section. The table shows the means and SEs of the gold particle densities for the two types of fiber on each of the seven grids, and in addition the neurotensin-immunoreactive bouton(s) that was present on the grid of spinal cord that was processed on the same droplets during the postembedding reaction.

munogold staining. For this reason, we have used the silverintensified gold method (Chan et al., 1990; Sesack and Pickel, 1992) to detect neurotensin, since this provides a particulate marker that does not appear to interfere with postembedding immunogold staining.

\section{Methodological considerations}

We have previously used the same antiserum to neurotensin with the $\mathrm{ABC}$ method, and shown that the distribution of immunostained profiles is very similar to that observed in other studies of neurotensin-LI in dorsal horn (Gibson et al., 1981; Hunt et al., 1981; Seybold and Elde, 1982; DiFiglia et al., 1984), with a dense plexus of fibers in laminae I and II, and many neuronal cell bodies located near the border between laminae II and III (Todd et al., 1992; Fig. 1). In the present study, immunostaining was restricted to the most superficial parts of the Vibratome sections, but again punctate staining was seen in laminae I and II and some cell bodies were visible in the ventral part of lamina II and the dorsal part of lamina III. This staining was abolished by preincubation of the antiserum with neurotensin peptide.

Although the electron microscopic sections contained a background scattering of silver particles, this could readily be distinguished from specific labeling by examining serial sections. The background staining is probably related to the use of $1 \%$ glutaraldehyde in the fixative (Kosaka et al., 1986), which was necessary in order to retain glutamate in the tissue. Specific labeling with silver particles was seen in axonal boutons throughout laminae I and II, and these resembled neurotensinimmunoreactive boutons observed in previous studies in the rat (Seybold and Maley, 1984) and monkey (DiFiglia et al., 1984), in that they contained many round agranular vesicles and, in most cases, few dense-cored vesicles. The common association of silver particles with dense-cored vesicles presumably reflects the presence of peptides within these vesicles (Merighi et al., 1989). DiFiglia et al. (1984) reported that neurotensin-immunoreactive boutons formed synapses that were exclusively asymmetrical, while Seybold and Maley (1984) found that some of the synapses were symmetrical and others asymmetrical. In the present study most of the synapses formed by immunoreactive boutons were asymmetrical, although some
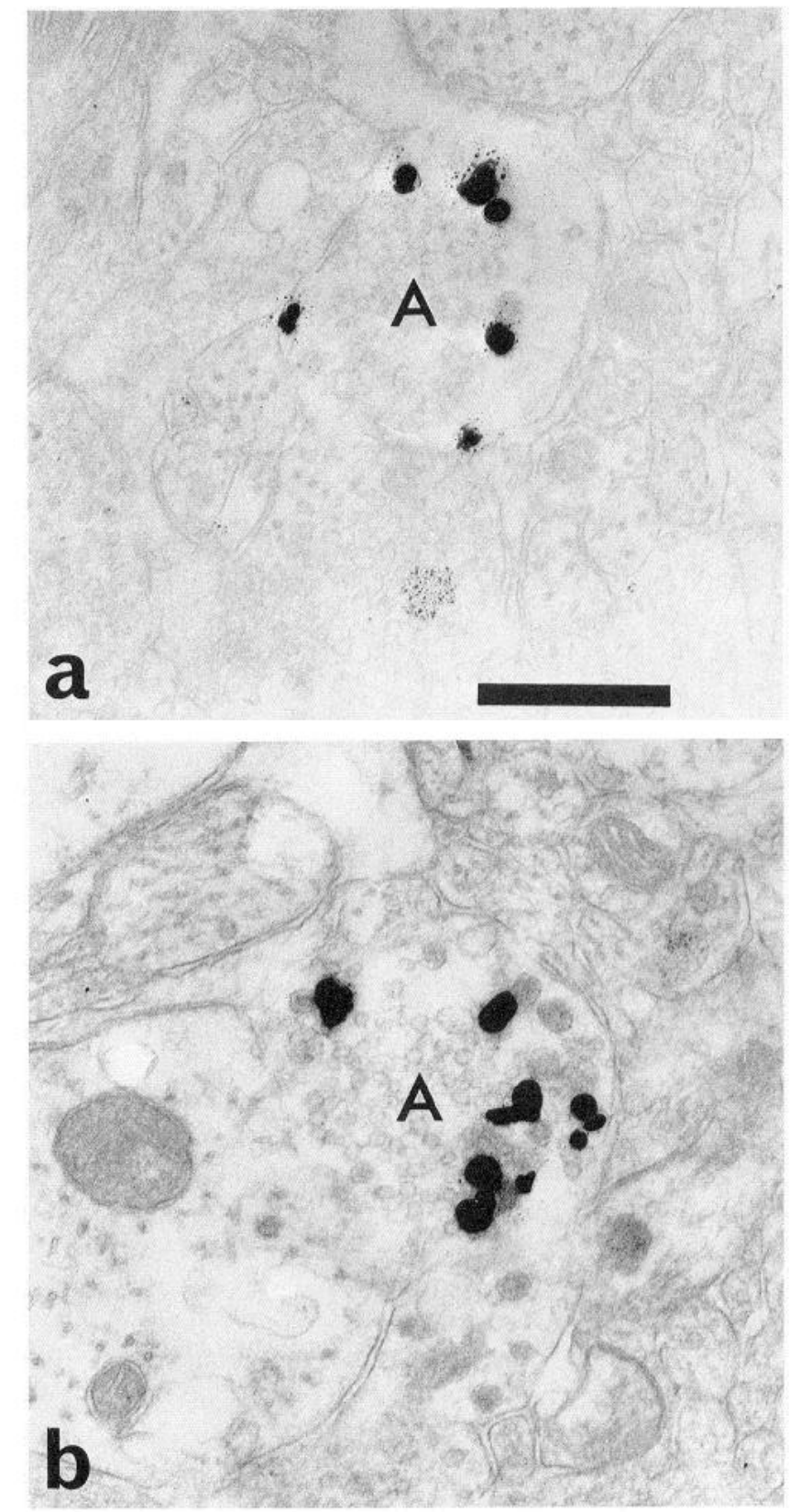

Figure 5. a, A neurotensin-immunoreactive axon $(A)$ from a section that had been processed by the postembedding method, but with omission of the glutamate antiserum. No $10 \mathrm{~nm}$ gold particles are present, indicating that the deposition of these particles is not related to the presence of neurotensin antibodies. $b$, Neurotensin-immunoreactive axon $(A)$ in a section treated with glutamate antiserum that had been incubated with glutamate conjugated to BSA. No $10 \mathrm{~nm}$ gold particles are present over this axon. Scale bar, $0.5 \mu \mathrm{m}$ for $a$ and $b$.

appeared to be symmetrical (Fig. 1d). The presence of a few symmetrical synapses may therefore represent a species difference between rat and monkey.

The similarity between the present results and previous reports of neurotensin immunoreactivity at both light and electron microscopic levels strongly suggests that the labeling method used in this study provided a reliable way of identifying neurotensin-containing axons. The failure of spinal hemisection or dorsal root section to produce a detectable alteration of neurotensin-LI in the superficial dorsal horn (Ninkovic et al., 1981; 
Yaksh et al., 1982) has been taken as evidence that neurotensinimmunoreactive axons in this region are derived from local cells. Even if a few of the neurotensin-immunoreactive axons that were observed in the present study arose from other sources (e.g., cells in deeper laminae), it is likely that at least the great majority were derived from neurotensin-containing neurons with cell bodies in laminae I-III.

The appearance of glutamate-LI seen here is also very similar to that reported previously in the spinal dorsal horn, with the labeling heaviest over axons that contained round vesicles and formed asymmetrical synapses, and light over axons with flattened vesicles that formed symmetrical synapses (Maxwell et al., 1990, 1992; Merighi et al., 1991; Westlund et al., 1992). In addition, immunostaining was almost completely abolished when glutamate conjugated to BSA had been added to the antiserum. Since the neurotensin antiserum was also raised in rabbit, it was necessary to establish that this was not detected by the secondary antibody used for the postembedding reaction. When the glutamate antiserum was omitted, no $10 \mathrm{~nm}$ gold particles were present on the sections (Fig. $5 a$ ), presumably because the neurotensin antibodies were sufficiently altered during the dehydration, osmication, and resin embedding of the Vibratome sections that they were not recognized by the anti-rabbit $\operatorname{IgG}$ coupled to $10 \mathrm{~nm}$ gold particles. All of the postembedding staining therefore presumably represents glutamate-LI.

In sections of central nervous tissue reacted with antisera to glutamate, gold particles are found at varying densities over most glial and neuronal profiles (Somogyi et al., 1986), and it is therefore necessary to quantify the particle density in order to determine whether glutamate-LI is enriched over particular structures. Even when different electron microscopic sections are reacted by the same protocol, there can be substantial differences in the absolute density of labeling (Ji ct al., 1991). This may be due to differences in the retention of glutamate in different animals during fixation, and also to inadvertent alterations in the conditions (e.g., ambient temperature) under which the reaction was carried out. For this reason, the gold particle density in each neurotensin-immunoreactive bouton was compared with that in nearby profiles from the same section, which formed asymmetrical or symmetrical synapses. The former group is likely to consist (at least mainly) of axons that use glutamate as a transmitter (Maxwell et al., 1990; Merighi et al., 1991), while those in the latter group are likely to use GABA, glycine, or ACh (McLaughlin et al., 1975; Barber et al., 1978; Ribeiroda-Silva and Cuello, 1990; Todd, 1990; Mitchell et al., 1993). Since gold particle densities were high over neurotensin-immunoreactive boutons, compared to other nearby structures, and were generally similar to those seen in boutons that formed asymmetrical synapses (Fig. 4), it is likely that glutamate is used as a transmitter by neurotensin-immunoreactive axons. The density in neurotensin-immunoreactive boutons was slightly (11.1\%) lower than that in the boutons that formed asymmetrical synapses and this difference was found to be significant. The latter group is likely to be heterogeneous, and may have included boutons of primary afferent, supraspinal and intrinsic origin. It may be that one or more of these populations consists of axons with a higher density of glutamate than that which is present in neurotensin-containing axons, in the same way that the level of glutamate-LI has been found to be significantly higher in mossy fiber terminals than in parallel fiber terminals in the cerebellum (Somogyi et al., 1986).

To provide further evidence, glutamate-LI in neurotensin- immunoreactive boutons was compared with that in cerebellar mossy and parallel fiber terminals. In order to minimize variation in immunostaining intensity, the cerebellar material had been subjected to the same preembedding method, and the sections were paired with sections of spinal cord and processed on the same droplets of reagents during the postembedding reaction. The gold particle densities over neurotensin-immunoreactive boutons were similar to those observed in parallel fiber terminals, but were significantly lower than those in mossy fiber terminals. Since both parallel and mossy fibers are thought to use glutamate as a transmitter (Sandoval and Cotman, 1978; Stone, 1979; Garthwaite and Brodbelt, 1990), the finding that cerebellar parallel fiber terminals and neurotensin-immunoreactive boutons in dorsal horn had similar levels of glutamateLI supports the conclusion that the latter also use glutamate as a transmitter.

\section{Neurotensin-containing cells in laminae I-III}

The present results therefore suggest that many, if not all, neurotensin-containing cells in the dorsal horn are excitatory, glutamatergic interneurons. Although many neuropeptides are present in neurons in laminae I-III of the dorsal horn, there is apparently no evidence for significant coexistence of peptides in cells that contain neurotensin. Somatostatin-LI is also present in neurons that do not contain GABA; however, somatostatin and neurotensin do not appear to coexist in axons within the dorsal horn, and are therefore presumably contained in different populations of cells (Proudlock et al., 1993). Neuropeptide Y appears to be present exclusively in GABAergic neurons (Rowan et al., 1993). We have recently used a double-labeling immunofluorescence technique to look for possible coexistence of neurotensin with several other peptides that are contained in spinal neurons: met-enkephalin, dynorphin, substance $\mathrm{P}$, and neurokinin B. Although neurotensin very occasionally coexisted with each of these peptides, the great majority of neurotensinimmunoreactive profiles did not show immunoreactivity with any of these peptide antisera (A. R. Brodbelt, A. J. Todd, and R. C. Spike, unpublished observations). It is therefore likely that the neurons that contain neurotensin are different from those that contain any of these other peptides. Yoshida et al. (1990) reported that $75 \%$ of neurotensin-immunoreactive neurons in the superficial dorsal horn were also immunoreactive with antiserum to the calcium-binding protein calbindin. Since Antal et al. (1991) found that some calbindin-immunoreactive neurons in laminae II and III accumulated ${ }^{3} \mathrm{H}-\mathrm{D}$-aspartate, this group may have included some neurotensin-containing cells.

Since these neurons are presumably capable of releasing both glutamate and neurotensin, and the two compounds may act on different targets, it is necessary to consider the function of the neurons in terms of the actions of both neurotensin and glutamate. The actions of neurotensin within the spinal cord are relatively poorly understood. When it is injected into the dorsal horn by iontophoresis, neurons may be excited (Miletic and Randic, 1979; Stanzione and Ziegelgansberger, 1983) or inhibited (Henry, 1982). If neurotensin is applied directly to the cord dorsum it causes expression of the proto-oncogene product $\mathrm{c}$-fos in a few neurons, and this is consistent with an excitatory action on these cells (Leah et al., 1989). When applied by intrathecal injection, neurotensin has an apparent analgesic action, although this depends on the test that is used (Yaksh et al., 1982; Spampinato et al., 1988).

The existence of excitatory interneurons in dorsal horn has 
been invoked in order to explain two aspects of the mismatch between dendritic trees of dorsal horn neurons and their receptive field properties. Many cells in deeper laminae have a major input from unmyelinated primary afferents but do not have dendritic trees that extend into laminae I or II (the major termination zone of these afferents; Sugiura et al., 1986), and it has been suggested that the inputs from these afferents are conveyed via excitatory interneurons with axons that pass ventrally (Ritz and Greenspan, 1985; Woolf and King, 1987). In addition, there is no clear relationship between the mediolateral and rostrocaudal spread of the dendritic tree of a neuron, and the size of its excitatory receptive field, and this suggests that much of the receptive field is due to polysynaptic inputs (Woolf, 1989). Since neurotensin-immunoreactive axons are largely restricted to the superficial dorsal horn, it is unlikely that neurotensincontaining neurons have significant axonal arborizations ventral to lamina II, and therefore they are not likely to be involved in relaying input from unmyelinated primary afferents to the deep dorsal horn. However, it is quite possible that they are involved in distributing information from primary afferents to other areas of the superficial dorsal horn, and are therefore partially responsible for enlarging the receptive fields of other dorsal horn neurons or strengthening their responses to peripheral stimulation.

It is difficult to use pharmacological techniques to study the actions of glutamate released from a particular population of spinal interneurons, because of the multiple origins of glutamatergic axons within the dorsal horn. In order to understand the actions of glutamate released by these neurons, it will be necessary to have further information about the postsynaptic targets of their axons and the types of excitatory amino acid receptor at which glutamate is acting. By using neurotensin antiscra as markcrs for the axons of these cells, it may be possible to obtain some of this information from immunocytochemical studies.

\section{References}

Antal M, Polgar E, Chalmers J, Minson JB, Lewellyn-Smith I, Heizmann CW, Somogyi $P$ (1991) Different populations of parvalbuminand calbindin-D28k-immunoreactive neurons contain GABA and accumulate ${ }^{3} \mathrm{H}$-D-aspartate in the dorsal horn of the rat spinal cord. J Comp Neurol 314:114-124.

Barber RP, Vaughn JE, Saito K, McLaughlin BJ, Roberts E (1978) GABAergic terminals are presynaptic to primary afferent terminals in the substantia gelatinosa of the rat spinal cord. Brain Res 141:3555 .

Barber RP, Vaughn JE, Roberts E (1982) The cytoarchitecture of GABAergic neurons in rat spinal cord. Brain Res 238:305-328.

Bennett GJ, Abdelmoumene M, Hayashi H, Dubner R (1980) Physiology and morphology of substantia gelatinosa neurons intracellularly stained with horseradish peroxidase. J Comp Neurol 194:809827.

Broman J, Ottersen OP (1992) Cervicothalamic tract terminals are enriched in glutamatc-likc immunorcactivity: an clectron microscopic double-labeling study in the cat. J Neurosci 12:204-221.

Carlton SM, Hayes ES (1990) Light microscopic and ultrastructural analysis of GABA-immunoreactive profiles in the monkey spinal cord. J Comp Neurol 300:162-182.

Chan J, Aoki C, Pickel VM (1990) Optimization of differential immunogold silver and peroxidase labeling with maintenance of ultrastructure in brain sections before plastic embedding. J Neurosci Methods 33:113-127.

DiFiglia M, Aronin N, Leeman SE (1984) Ultrastructural localization of immunoreactive neurotensin in the monkey superficial dorsal horn. J Comp Neurol 225:1-12.

Fonnum F (1984) Glutamate: a neurotransmitter in mammalian brain. J Neurochem 46:1-11.
Garthwaite J, Brodbelt AR (1990) Glutamate as the principal mossy fibre transmitter in rat cerebellum: pharmacological evidence. Eur $\mathbf{J}$ Neurosci 2:177-180.

Gibson SJ, Polak J, Bloom SR, Wall PD (1981) The distribution of nine peptides in rat spinal cord with special emphasis on the substantia gelatinosa and on the area around the central canal (lamina X). J Comp Neurol 201:65-80.

Henry JL (1982) Electrophysiological studies on the neuroactive properties of neurotensin. Ann NY Acad Sci 400:216-227.

Holstege JC (1991) Ultrastructural evidence for GABAergic brain stem projections to spinal motoneurons in the rat. J Neurosci 11:159-167.

Hunt SP, Kelly JS, Emson PC, Kimmel JR, Miller RJ, Wu J-Y (1981) An immunohistochemical study of neuronal populations containing neuropeptides or $\gamma$-aminobutyrate within the superficial layers of the rat dorsal horn. Neuroscience 6:1883-1898.

Jennes L, Stumpf WE, Kalivas PW (1982) Neurotensin: topographical distribution in rat brain by immunohistochemistry. J Comp Neurol 210:211-224.

Ji Z, Aas J-E, Laake J, Walberg F, Ottersen OP (1991) An electron microscopic, immunogold analysis of glutamate and glutamine in terminals of rat spinocerebellar fibers. J Comp Neurol 307:296-310.

Kosaka T, Nagatsu I, Wu JY, Hama K (1986) Use of high concentrations of glutaraldehyde for immunocytochemistry of transmitter synthesizing enzymes in the central nervous system. Neuroscience 19: 975-990.

Leah J, Menetrey D, de Pommery J (1988) Neuropeptides in long ascending spinal tract cells in the rat: evidence for parallel processing of ascending information. Neuroscience 24:195-207.

Lcah J, Herdegen T, Zimmerman M (1989) Physiological and pharmacological induction of $c$-fos protein immunoreactivity in superficial dorsal horn neurones. In: Processing of sensory information in the superficial dorsal horn of the spinal cord (Cervero F, Bennett GJ, Headley PM, eds), pp 307-310. New York: Plenum.

Light AR, Kavookjian AM (1988) Morphology and ultrastructure of physiologically identified substantia gelatinosa (lamina II) neurons with axons that terminate in deeper dorsal horn laminae (III-V). J Comp Neurol 267:172-189.

Magoul R, Onteniente B, Geffard M, Calas A (1987) Anatomical distribution and ultrastructural organization of the GABAergic system in the rat spinal cord. An immunocytochemical study using antiGABA antibodies. Neuroscience 20:1001-1009.

Maxwell DJ, Christie WM, Short AD, Storm-Mathisen J, Ottersen OP (1990) Central boutons of glomeruli in the spinal cord of the cat arc enriched with L-glutamate-like immunoreactivity. Neuroscience 36 : 83-104.

Maxwell DJ, Christie WM, Short AD, Brown AG (1991) Direct observations of synapses between GABA-immunoreactive boutons and identified spinocervical tract neurons in the cat's spinal cord. J Comp Neurol 307:375-392.

Maxwell DJ, Christie WM, Brown AG, Ottersen OP, Storm-Mathisen J (1992) Direct observations of synapses between L-glutamate-immunoreactive boutons and identified spinocervical tract neurones in the spinal cord of the cat. J Comp Neurol 326:485-500.

McLaughlin BJ, Barber R, Saito K, Roberts E, Wu J-Y (1975) Immunocytochemical localization of glutamate decarboxylase in rat spinal cord. J Comp Neurol 164:305-322.

Mcrighi A, Polak JM, Fumagalli G, Thcodosis DT (1989) Ultrastructural localization of neuropeptides and GABA in rat dorsal horn: a comparison of different immunogold labeling techniques. J Histochem Cytochem 37:529-540.

Merighi A, Polak JM, Theodosis DT (1991) Ultrastructural visualization of glutamate and aspartate immunoreactivities in the rat spinal dorsal horn, with special reference to the co-localization of glutamate, substance $P$ and calcitonin-gene related peptide. Neuroscience 40:6780 .

Miletic V, Randic M (1979) Neurotensin excites cat spinal neurons located in laminae I-III. Brain Res 169:600-604.

Miller KE, Clements JR, Larson AA, Beitz AJ (1988) Organization of glutamate-like immunoreactivity in the rat superficial dorsal horn: light and electron microscopic observations. Synapse 2:28-36.

Mitchell K, Spike RC, Todd AJ (1993) An immunocytochemical study of glycine receptor and GABA in laminae I-III of rat spinal dorsal horn. J Neurosci 13:2371-2381.

Montero VM, Wenthold RJ (1989) Quantitative immunogold analysis reveals high glutamate levels in retinal and cortical synaptic terminals 
in the lateral geniculate nucleus of the macaque. Neuroscience 31 : 639-647.

Nandi KN, Knight DS, Beal JA (1993) Spinal neurogenesis and axon projection: a correlative study in the rat. J Comp Neurol 328:252262.

Ninkovic M, Hunt SP, Kelly JS (1981) Effect of dorsal rhizotomy on the autoradiographic distribution of opiate and neurotensin receptors and neurotensin-like immunoreactivity within the rat spinal cord. Brain Res 230:111-119.

Ottersen OP (1989) Quantitative electron microscopic immunocytochemistry of neuroactive amino acids. Anat Embryol (Berl) 180:115.

Ottersen OP, Storm-Mathisen J (1984) Glutamate- and GABA-containing neurons in the mouse and rat brain, as demonstrated with a new immunocytochemical technique. J Comp Neurol 229:374-392.

Proudlock F, Spike RC, Todd AJ (1993) Immunocytochemical study of somatostatin, neurotensin, GABA, and glycine in rat spinal dorsal horn. J Comp Neurol 327:289-297.

Rexed B (1952) The cytoarchitectonic organization of the spinal cord in the cat. J Comp Neurol 96:415-495.

Ribeiro-da-Silva A, Cuello AC (1990) Choline acetyltransferase-immunoreactive profiles are presynaptic to primary sensory fibres in the rat superficial dorsal horn. J Comp Neurol 295:370-384.

Ritz LA, Greenspan JD (1985) Morphological features of lamina V neurons receiving nociceptive input in cat sacrocaudal spinal cord. $J$ Comp Neurol 238:440-452.

Rowan S, Todd AJ, Spike RC (1993) Evidence that neuropeptide Y is present in GABAergic neurons in the superficial dorsal horn of the rat spinal cord. Neuroscience 53:537-545.

Rustioni A, Cuenod M (1982) Selective retrograde transport of D-aspartate in spinal interneurons and cortical neurons of rats. Brain Res 236:143-155.

Sandoval ME, Cotman CW (1978) Evaluation of glutamate as a neurotransmitter of cerebellar parallel fibers. Neuroscience 3:199-206.

Sesack SR, Pickel VM (1992) Dual ultrastructural localization of enkephalin and tyrosine hydroxylase immunoreactivity in the rat ventral tegmental area: multiple substrates for opiate dopamine interaction. J Neurosci 12:1335-1350.

Seybold V, Elde R (1982) Neurotensin immunoreactivity in the superficial laminae of the dorsal horn of the rat. I. Light microscopic studies of cell bodies and proximal dendrites. J Comp Neurol 205: $89-100$.

Seybold V, Maley B (1984) Ultrastructural study of neurotensin immunoreactivity in the superficial laminae of the dorsal horn of the rat. Peptides 5:1179-1189.

Somogyi P, Halasy K, Somogyi J, Storm-Mathisen J, Ottersen OP (1986) Quantification of immunogold labelling reveals enrichment of glutamate in mossy and parallel fibre terminals in cat cerebellum. Neuroscience 19:1045-1050.

Spampinato S, Romualdi P, Candaletti S, Cavacchini E, Ferri S (1988) Distinguishable effects of intrathecal dynorphins, somatostatin, neurotensin, and s-calcitonin on nociception and motor function in the rat. Pain 35:95-104.

Spike RC, Todd AJ (1992) An ultrastructural and immunocytochemical study of lamina II islet cells in rat spinal dorsal horn. J Comp Neurol 323:359-369.

Stanzione P, Ziegelgansberger W (1983) Action of neurotensin on spinal cord neurons in the rat. Brain Res 268:111-118.
Stone TW (1979) Glutamate as the neurotransmitter of cerebellar granule cells in the rat: electrophysiological evidence. Br J Pharmacol 66:291-296.

Storm-Mathisen J, Leknes AK, Bore A, Vaaland JL, Edminson P, Haug FMS, Ottersen OP (1983) First visualisation of glutamate and GABA in neurons by immunocytochemistry. Nature 301:517-520.

Streit $P$ (1980) Selective retrograde labeling indicating the transmitter of neuronal pathways. J Comp Neurol 191:429-463.

Sugiura Y, Lee CL, Perl ER (1986) Central projections of identified unmyelinated (C) afferent fibers innervating mammalian hairy skin. Science 234:358-361.

Todd AJ (1990) An electron microscope study of glycine-like immunoreactivity in laminae I-III of the spinal dorsal horn of the rat. Neuroscience 39:387-394.

Todd AJ (1991) Immunohistochemical evidence that acetylcholinc and glycine exist in different populations of GABAergic neurons in lamina III of rat spinal dorsal horn. Neuroscience 44:741-746.

Todd AJ, McKenzie J (1989) GABA-immunoreactive neurons in the dorsal horn of the rat spinal cord. Neuroscience 31:799-806.

Todd AJ, Sullivan AC (1990) Light microscope study of GABA-like and glycine-like immunoreactivities in the spinal cord of the rat. $J$ Comp Neurol 296:496-505.

Todd AJ, Russell G, Spike RC (1992) Immunocytochemical evidence that GABA and neurotensin exist in different neurons in laminae II and III of rat spinal dorsal horn. Neuroscience 47:685-691.

Walberg F, Ottersen OP, Rinvik E (1990) GABA, glycine, aspartate, glutamate and taurine in the vestibular nuclei: an immunocytochemical investigation in the cat. Exp Brain Res 79:547-563.

Weinberg RJ, Conti F, Van Eyck SL, Pctrusz P, Rustioni A (1987) Glutamate immunoreactivity in superficial laminae of rat dorsal horn and spinal trigeminal nucleus. In: Neurology and neurobiology, Vol 24, Excitatory amino acid transmission (Hicks TP, Lodge D, McLennan H, eds), pp 173-176. New York: Liss.

Westlund KN, Carlton SM, Zhang D, Willis WD (1992) Glutamateimmunoreactive terminals synapse on primate spinothalamic tract cells. J Comp Neurol 322:519-527.

Woolf CJ (1989) Afferent induced alterations of receptive field properties. In: Processing of sensory information in the superficial dorsal horn of the spinal cord (Cervero F, Bennett GJ, Headley PM, eds), pp 443-462. New York: Plenum.

Woolf CJ, King AE (1987) Physiology and morphology of multireceptive neurons with $\mathrm{C}$-afferent fibre inputs in the deep dorsal horn of the rat lumbar spinal cord. J Neurophysiol 58:105-119.

Yaksh TL, Schmauss C, Micevych PE, Abay EO, Go VLW (1982) Pharmacological studies on the application, disposition and release of neurotensin in the spinal cord. Ann NY Acad Sci 400:228-243.

Yingcharoen K, Rinvik E, Storm-Mathisen J, Ottersen OP (1989) GABA, glycine, glutamate, aspartate and taurine in the perihypoglossal nuclei: an immunocytochemical investigation in the cat with particular reference to the issue of amino acid colocalization. Exp Brain Res 78:345-357.

Yoshida S, Senba E, Kubota Y, Hagihira S, Yoshiya I, Emson PC, Tohyama $M$ (1990) Calcium-binding proteins calbindin and parvalbumin in the superficial dorsal horn of the rat spinal cord. Neuroscience $37: 839-848$. 